\title{
Amplitude Variation with Angle Inversion for New Parameterized Porosity and Fluid Bulk Modulus
}

\author{
Shengjun Li $\mathbb{D}^{1,2}$ Bingyang Liu $\mathbb{D}^{1},{ }^{1}$ Jianhu Gao $\mathbb{D}^{1},{ }^{1}$ and Huaizhen Chen $\mathbb{D}^{3}$ \\ ${ }^{1}$ Research Institute of Petroleum Exploration and Development-Northwest, PetroChina, Lanzhou 730020, China \\ ${ }^{2}$ State Key Laboratory of Petroleum Resources and Prospecting, China University of Petroleum, Beijing 102202, China \\ ${ }^{3}$ State Key Laboratory of Marine Geology, School of Ocean and Earth Science, Institute for Advanced Study, Tongji University, \\ Shanghai 200092, China
}

Correspondence should be addressed to Huaizhen Chen; chenhz2011@gmail.com

Received 31 August 2020; Revised 25 December 2020; Accepted 30 December 2020; Published 13 January 2021

Academic Editor: Yuanyuan Zha

Copyright (c) 2021 Shengjun Li et al. This is an open access article distributed under the Creative Commons Attribution License, which permits unrestricted use, distribution, and reproduction in any medium, provided the original work is properly cited.

Estimating porosity and fluid bulk modulus is an important goal of reservoir characterization. Based on the model of fluid substitution, we first propose a simplified bulk modulus of a saturated rock as a function of bulk moduli of minerals and fluids, in which we employ an empirical relationship to replace the bulk modulus of dry rock with that of minerals and a new parameterized porosity. Using the simplified bulk modulus, we derive a PP-wave reflection coefficient in terms of the new parameterized porosity and fluid bulk modulus. Focusing on reservoirs embedded in rocks whose lithologies are similar, we further simplify the derived reflection coefficient and present elastic impedance that is related to porosity and fluid bulk modulus. Based on the presented elastic impedance, we establish an approach of employing seismic amplitude variation with offset/angle to estimate density, new parameterized porosity, and fluid bulk modulus. We finally employ noisy synthetic seismic data and real datasets to verify the stability and reliability of the proposed inversion approach. Test on synthetic seismic data illustrates that the proposed inversion approach can produce stable inversion results in the case of signal-to-noise ratio (SNR) of 2 , and applying the approach to real datasets, we conclude that reliably results of porosity and fluid bulk modulus are obtained, which is useful for fluid identification and reservoir characterization.

\section{Introduction}

Identification of fluid type and prediction of porosity are important targets for reservoir characterization. An effective model of fluid substitution proposed by Gassmann [1] is useful for computing bulk modulus of saturated rock in the case of different values of saturation and porosity. Based on the fluid substitution model, many studies focus on employing prestacked seismic amplitude to implement the inversion for fluid indicator and porosity.

To estimate different properties of hydrocarbon reservoirs from seismic data, reflection coefficients of seismic wave are derived. Aki and Richards [2] propose reflection coefficients of PP, PS, SS, and SP waves as a function of Pand $\mathrm{S}$-wave reflectivities in the case of an interface separating two isotropic media. Following Aki and Richards [2], many different reflection coefficients expressed as reflectivities of impedances, Lamé constants, moduli, and fluid indicators [3-10] are proposed. Similar to acoustic impedance (AI), Connolly [11] presents an expression of elastic impedance (EI) that varies with the incidence angle. Based on the parameterized EI, partially incidence angle-stacked seismic data are employed to estimate EI datasets, and then, parameters of reservoir properties are extracted from the estimated EI [4, 10].

Based on the derived reflection coefficient and EI, different inversion approaches are established for employing amplitude variation with offset/angle (AVO/AVA) to estimate elastic parameters (e.g., P- and S-wave velocities, moduli, and density) and reservoir parameters (e.g., porosity, clay volume, and water saturation). Following the Bayesian framework, geophysicists implement inversion of AVO/AVA data for unknown parameters involving elastic and reservoir parameters $[7,8,10,12]$. The two-step 
inversion approach, which involves the inversion of AVO/AVA data for EI datasets and the estimation of elastic parameters from inverted EI datasets, is well employed in hydrocarbon reservoir exploration and characterization. To estimate unknown parameters from inverted EI datasets, Chen et al. [3] present an approach of utilizing both firstand second-order derivatives of EI with respect to unknown parameter vector to improve the accuracy of unknown parameter estimate.

In the present study, we first employ an empirical relationship given by Krief et al. [13] to rewrite dry rock bulk modulus and we also present a new parameterized porosity. Using the fluid substitution model, we present a simplified bulk modulus of saturated rock as a function of the new parameterized porosity and fluid bulk modulus, and then, we derive a linearized reflection coefficient in terms of reflectivities of bulk and shear moduli of minerals, density, new parameterized porosity, and fluid bulk modulus. Focusing on gas-bearing reservoirs embedded in layers with similar lithologies, we neglect the effects of minerals on the derived reflection coefficient and propose a new expression of EI. Based on the reflection coefficient and EI, we establish a two-step inversion approach involving (1) inversion of prestacked seismic data for EI and (2) estimation of new parameterized porosity and fluid bulk modulus from the inverted EI. Synthetic and real datasets are utilized to verify the stability and reliability of the inversion approach.

\section{Theory and Method}

2.1. A New Parameterized PP-Wave Reflection Coefficient. Using the fluid substitution equations proposed by Gassmann [1], we compute the bulk modulus of a saturated rock as a function of the bulk moduli of dry rock and fluids

$$
K_{\text {sat }}=K_{\text {dry }}+\frac{\left(1-K_{\mathrm{dry}} / K_{0}\right)^{2}}{\phi / K_{\mathrm{f}}+(1-\phi) / K_{0}-K_{\mathrm{dry}} / K_{0}^{2}},
$$

where $K_{\text {sat }}$ is the bulk modulus of saturated rock, $K_{\text {dry }}$ is the bulk modulus of dry rock, $K_{\mathrm{f}}$ is the fluid bulk modulus, $K_{0}$ is the bulk modulus of minerals making up the rock, and $\phi$ is the rock porosity, respectively.

We next employ empirical relationships between the bulk and shear moduli of minerals and dry rock (i.e., $K_{\text {dry }}$ and $K_{0}$ ) proposed by Krief et al. [13]

$$
\begin{gathered}
K_{\mathrm{dry}}=K_{0}(1-\phi)^{3 /(1-\phi)}, \\
\mu_{\mathrm{dry}}=\mu_{0}(1-\phi)^{3 /(1-\phi)},
\end{gathered}
$$

where $\mu_{0}$ and $\mu_{\text {dry }}$ are shear moduli of minerals and dry rock, respectively, to simplify equation (1) as

$$
K_{\mathrm{sat}}=K_{\mathrm{dry}}+\frac{\left(1-(1-\phi)^{3 /(1-\phi)}\right)^{2}}{\phi / K_{\mathrm{f}}+(1-\phi) / K_{0}-(1-\phi)^{3 /(1-\phi)} / K_{0}} .
$$

Under the assumption of $K_{\mathrm{f}} \ll K_{0}$ in the case of gasbearing reservoirs, we further simplify the bulk modulus of saturated rock as

$$
K_{\text {sat }}=K_{\text {dry }}+\phi_{\mathrm{n}} K_{\mathrm{f}}
$$

where $\phi_{\mathrm{n}}=\left(1-(1-\phi)^{3 /(1-\phi)}\right)^{2} / \phi$ is a new parameterized porosity. In Figure 1, we plot how the new parameterized porosity $\phi_{\mathrm{n}}$ varies with the porosity $\phi$. We observe that $\phi_{\mathrm{n}}$ monotonously increases with $\phi$. Compared with the porosity $\phi$ itself, the value of $\phi_{\mathrm{n}}$ is much larger than that of $\phi$ and has a wider range. We stress that the new parameterized porosity $\phi_{\mathrm{n}}$ is dimensionless which is the same to the porosity $\phi$. However, the effect of porosity is still coupled with that of fluid bulk modulus in the simplified bulk modulus of saturated rock.

Following Gassmann [1], we also assume the shear modulus of saturated rock is equal to that of dry rock, which is given by

$$
\mu=\mu_{\mathrm{sat}}=\mu_{\mathrm{dry}}
$$

where $\mu_{\text {sat }}$ is the shear modulus of saturated rock.

Using the simplified bulk and shear moduli of saturated rock, we next express P-wave modulus $M_{\text {sat }}$ as

$$
M_{\text {sat }}=M_{\text {dry }}+\phi_{\mathrm{n}} K_{\mathrm{f}}
$$

where $M_{\text {dry }}=K_{\text {dry }}+(4 / 3) \mu_{\text {dry }}$.

Following Downton [12] and Zong et al. [10], we express PP-wave reflection coefficient in terms of bulk and shear moduli of dry rock, the new parameterized porosity and the fluid bulk modulus

$$
\begin{aligned}
R_{\mathrm{PP}}(\theta)= & \frac{1}{4} \frac{g_{\text {sat }}}{g_{\text {dry }}} \sec ^{2} \theta \frac{\Delta M_{\text {dry }}}{M_{\text {dry }}}+\frac{1}{4}\left(1-\frac{g_{\text {sat }}}{g_{\text {dry }}}\right) \sec ^{2} \theta \frac{\Delta \phi_{\mathrm{n}}}{\phi_{\mathrm{n}}} \\
& +\frac{1}{4}\left(1-\frac{g_{\text {sat }}}{g_{\text {dry }}}\right) \sec ^{2} \theta \frac{\Delta K_{\mathrm{f}}}{K_{\mathrm{f}}}-2 g_{\text {sat }} \sin ^{2} \theta \frac{\Delta \mu}{\mu} \\
& +\left(\frac{1}{2}-\frac{1}{4} \sec ^{2} \theta\right) \frac{\Delta \rho}{\rho},
\end{aligned}
$$

where $\theta$ is the incidence angle, $g_{\text {sat }}=\mu / M_{\text {sat }}$, and $g_{\text {dry }}=\mu /$ $M_{\text {dry }}$.

In the derived PP-wave reflection coefficient, we observe that effects of dry rock modulus, porosity, and fluid bulk modulus on reflection coefficient are separated, which may guide us to employ seismic reflection amplitudes to predict fluid type and reservoir property. However, we also see that parameters related to the incidence angle $\theta$ before the reflectivities $\Delta \phi_{\mathrm{n}}$ / $\phi_{\mathrm{n}}$ and $\Delta K_{\mathrm{f}} / K_{\mathrm{f}}$ are the same, which may induce errors in the estimation of porosity and fluid bulk modulus using datasets of amplitude variation with offset/angle (AVO/AVA). 


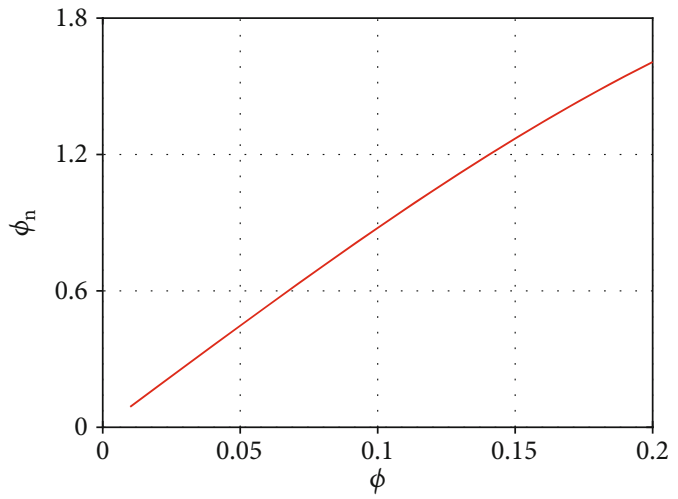

FIGURE 1: Variation of new parameterized porosity $\phi_{\mathrm{n}}$ with the porosity $\phi$.

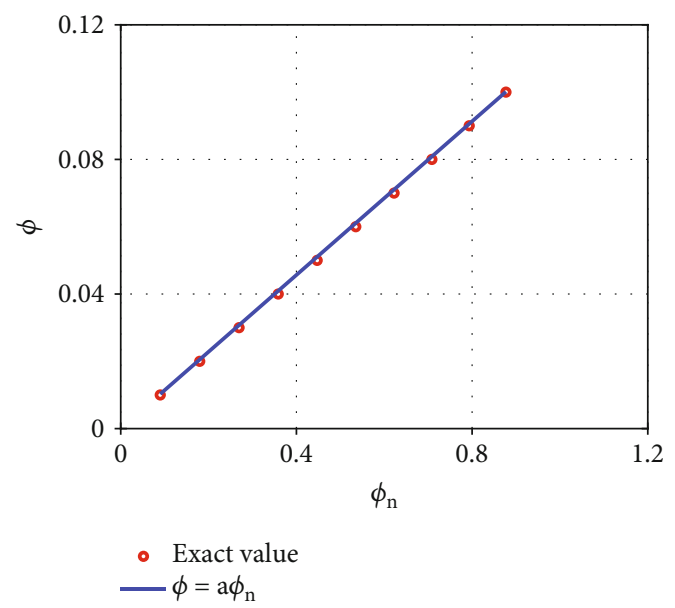

Figure 2: Comparison between the exact value of $\phi$ and the approximate result computed using the linear relationship. The variable $a$ is obtained from the linear fit between $\phi$ and $\phi_{\mathrm{n}}$, and $a$ $=0.1141$.

We next rewrite $\mathrm{P}$ - and S-wave moduli of dry rock as a function of the new parameterized porosity $\phi_{\mathrm{n}}$

$$
\begin{gathered}
M_{\mathrm{dry}}=M_{0}\left(1-\sqrt{\phi_{\mathrm{n}} \phi}\right), \\
\mu_{\mathrm{dry}}=\mu_{0}\left(1-\sqrt{\phi_{\mathrm{n}} \phi}\right),
\end{gathered}
$$

where $M_{0}$ and $\mu_{0}$ are $\mathrm{P}$ - and S-wave moduli of minerals making up the rock, respectively. In the case of $\phi \leq 0.1$, we employ an approximately linear relationship between $\phi_{\mathrm{n}}$ and $\phi$, which is given by

$$
\phi \approx a \phi_{\mathrm{n}}
$$

where $a$ is obtained from the linear fit between $\phi$ and $\phi_{n}$ shown in Figure 2, to further rewrite the derived reflection coefficient. In Figure 2, we observe there is a good match between the new parameterized porosity $\phi_{\mathrm{n}}$ computed using the nonlinear relationship, i.e., $\phi_{\mathrm{n}}=\left(1-(1-\phi)^{3 /(1-\phi)}\right)^{2} / \phi$, and that calculated using the approximately linear relationship (equation (9)) in the case of $\phi \leq 0.1$.

Substituting equations (8) and (9) into equation (7), we obtain the reexpressed $\mathrm{PP}$-wave reflection coefficient as

$$
\begin{aligned}
R_{\mathrm{PP}}(\theta) \approx & p_{M}(\theta) \frac{\Delta M_{0}}{M_{0}}+p_{\mu}(\theta) \frac{\Delta \mu_{0}}{\mu_{0}}+p_{\rho}(\theta) \frac{\Delta \rho}{\rho}+p_{\phi_{\mathrm{n}}}(\theta) \frac{\Delta \phi_{\mathrm{n}}}{\phi_{\mathrm{n}}} \\
& +\mathrm{q}_{\phi_{\mathrm{n}}} \frac{\Delta \phi_{\mathrm{n}}}{1-\sqrt{\mathrm{a}} \phi_{\mathrm{n}}}+p_{K_{\mathrm{f}}}(\theta) \frac{\Delta K_{\mathrm{f}}}{K_{\mathrm{f}}}
\end{aligned}
$$

where

$$
\begin{aligned}
& p_{M}(\theta)=\frac{1}{4} \frac{g_{\text {sat }}}{g_{\text {dry }}} \sec ^{2} \theta, \\
& p_{\mu}(\theta)=-2 g_{\text {sat }} \sin ^{2} \theta, \\
& p_{\rho}(\theta)=\frac{1}{2}-\frac{1}{4} \sec ^{2} \theta, \\
& p_{\phi_{\mathrm{n}}}(\theta)=\frac{1}{4}\left(1-\frac{g_{\text {sat }}}{g_{\text {dry }}}\right) \sec ^{2} \theta, \\
& q_{\phi_{\mathrm{n}}}(\theta)=-\frac{\sqrt{a}}{4} \frac{g_{\text {sat }}}{g_{\text {dry }}} \sec ^{2} \theta+2 \sqrt{a} g_{\text {sat }} \sin ^{2} \theta, \\
& p_{K_{\mathrm{f}}}(\theta)=\frac{1}{4}\left(1-\frac{g_{\text {sat }}}{g_{\text {dry }}}\right) \sec ^{2} \theta .
\end{aligned}
$$

In equation (10), we observe that the reexpressed PP-wave reflection coefficient is a function of reflectivities of $\mathrm{P}$ - and $\mathrm{S}$ wave moduli of minerals, density, new parameterized porosity, and fluid bulk modulus, and there is also one term that is related to changes in new parameterized porosity (i.e., $\Delta \phi_{\mathrm{n}}$ ). Although the angle-dependent parameters before $\Delta \phi_{\mathrm{n}} / \phi_{\mathrm{n}}$ and $\Delta K_{\mathrm{f}} / K_{\mathrm{f}}$ are still the same, the parameter before the term $\Delta \phi_{\mathrm{n}}$ may change the feature of reflection coefficient varying with the incidence angle, which may help to decouple the influences of porosity $\phi_{\mathrm{n}}$ and fluid bulk modulus $K_{\mathrm{f}}$ on the reflection coefficient.

2.2. AVA Inversion for Fluid Bulk Modulus and New Parameterized Porosity. Based on the reexpressed reflection coefficient, we may implement the inversion for unknown parameters $\left(M_{0}, \mu_{0}, \rho, \phi_{\mathrm{n}}\right.$, and $\left.K_{\mathrm{f}}\right)$ using AVA datasets. Focusing on the case of reservoirs embedded in rocks with small changes in lithology, which means changes in P- and $\mathrm{S}$-wave moduli of minerals are small across the reflection interface, we may neglect the effects of reflectivities of Pand S-wave moduli of minerals on the reflection coefficients, and the reflection coefficient is further simplified as

$$
\begin{aligned}
R_{\mathrm{PP}}(\theta) \approx & p_{\rho}(\theta) \frac{\Delta \rho}{\rho}+p_{\phi_{\mathrm{n}}}(\theta) \frac{\Delta \phi_{\mathrm{n}}}{\phi_{\mathrm{n}}}+q_{\phi_{\mathrm{n}}}(\theta) \\
& \cdot \frac{\Delta \phi_{\mathrm{n}}}{1-\sqrt{\mathrm{a}} \phi_{\mathrm{n}}}+p_{K_{\mathrm{f}}}(\theta) \frac{\Delta K_{\mathrm{f}}}{K_{\mathrm{f}}}
\end{aligned}
$$


In equation (12), we observe the reflectivities of density, new parameterized porosity, and fluid bulk modulus and perturbations in new porosity are reserved. It means in the case of reservoirs with small changes in lithology, we solely need to invert for $\rho, \phi_{\mathrm{n}}$, and $K_{\mathrm{f}}$ using the AVA datasets.

Following Chen et al. [4], we extend the simplified reflection coefficient to be a time-continuous function

$$
\begin{aligned}
R_{\mathrm{PP}}(t, \theta)= & p_{\rho}(t, \theta) \frac{\partial}{\partial t} \ln \rho(t)+p_{\phi_{\mathrm{n}}}(t, \theta) \frac{\partial}{\partial t} \ln \phi_{\mathrm{n}}(t) \\
& +q_{\phi_{\mathrm{n}}}(t, \theta) \frac{1}{-\sqrt{a}} \frac{\partial}{\partial t} \ln \left(1-\sqrt{a} \phi_{\mathrm{n}}(t)\right) \\
& +p_{K_{\mathrm{f}}}(t, \theta) \frac{\partial}{\partial t} \ln K_{\mathrm{f}}(t),
\end{aligned}
$$

where $p_{\rho}(t, \theta), p_{\phi_{\mathrm{n}}}(t, \theta), q_{\phi_{\mathrm{n}}}(t, \theta)$, and $p_{K_{\mathrm{f}}}(t, \theta)$ are the timeand angle-dependent parameters, which are given by

$$
\begin{aligned}
& p_{\rho}(t, \theta)=\frac{1}{2}-\frac{1}{4} \sec ^{2} \theta, \\
& p_{\phi_{\mathrm{n}}}(t, \theta)=\frac{1}{4}\left[1-\frac{g_{\mathrm{sat}}(t)}{g_{\mathrm{dry}}(t)}\right] \sec ^{2} \theta, \\
& q_{\phi_{\mathrm{n}}}(t, \theta)=-\frac{\sqrt{a}}{4} \frac{g_{\text {sat }}(t)}{g_{\mathrm{dry}}(t)} \sec ^{2} \theta+2 \sqrt{a} g_{\mathrm{sat}}(t) \sin ^{2} \theta, \\
& p_{K_{\mathrm{f}}}(t, \theta)=\frac{1}{4}\left[1-\frac{g_{\text {sat }}(t)}{g_{\mathrm{dry}}(t)}\right] \sec ^{2} \theta,
\end{aligned}
$$

in which $g_{\text {sat }}(t)$ and $g_{\text {dry }}(t)$ are time-dependent S-to-P modulus ratios of saturated and dry rocks, respectively. In elastic impedance (EI) proposed by Connolly [11], the relationship between reflection coefficient and EI is given by

$$
R_{\mathrm{PP}}(t, \theta)=\frac{1}{2} \frac{\partial}{\partial t} \ln \mathrm{EI}(t, \theta)
$$

Combining equations (13) and (15) and taking an integral, we obtain the expression of logarithmic EI as

$$
\begin{aligned}
\operatorname{lnEI}(t, \theta)= & 2 p_{\rho}(t, \theta) \ln \rho(t)+2 p_{\phi_{\mathrm{n}}}(t, \theta) \ln \phi_{\mathrm{n}}(t) \\
& +2 q_{\phi_{\mathrm{n}}}(t, \theta) \frac{1}{-\sqrt{a}} \ln \left(1-\sqrt{a} \phi_{\mathrm{n}}(t)\right) \\
& +2 p_{K_{\mathrm{f}}}(t, \theta) \ln K_{\mathrm{f}}(t),
\end{aligned}
$$

and the expression of EI that solely considers the effects of density, porosity, and fluid bulk modulus is given by

$$
\begin{aligned}
\mathrm{EI}(t, \theta)= & \rho(t)^{2 p_{\rho}(t, \theta)} \phi_{\mathrm{n}}(t)^{2 p_{\phi_{\mathrm{n}}}(t, \theta)} K_{\mathrm{f}}(t)^{2 p_{K_{\mathrm{f}}}(t, \theta)} \\
& \cdot\left(1-\sqrt{a} \phi_{\mathrm{n}}(t)\right)^{2\left(q_{\phi_{\mathrm{n}}}(t, \theta) /-\sqrt{a}\right)} .
\end{aligned}
$$

Combining equations (16) and (17), we observe that using EI results estimated from prestacked seismic data, we may estimate density $\rho$, porosity $\phi_{\mathrm{n}}$, and fluid bulk modulus
TABLE 1: The clay volume, porosity, and water saturation of twolayer model.

\begin{tabular}{lccc}
\hline & $V_{\mathrm{c}}$ & $\phi$ & $S_{\mathrm{w}}$ \\
\hline Layer 1 & 0.1 & 0.03 & 1 \\
Layer 2 & 0.11 & 0.08 & 0.1 \\
\hline
\end{tabular}

$K_{\mathrm{f}}$, which may provide useful information for reservoir characterization and fluid identification. Following Chen et al. [3], we employ a least-squares (LS) algorithm to implement the first-step inversion for estimating EI datasets using the prestacked seismic data. To estimate density, new parameterized porosity, and fluid bulk modulus, we implement the second-step inversion, i.e., using the estimated EI results to predict the unknown parameter vector $\mathbf{m}$ involving $\rho, \phi_{n}$, and $K_{\mathrm{f}}$ based on the calculation of first- and second-order derivatives of EI with respect to $\mathbf{m}$. The first- and secondorder derivatives of EI with respect to $\mathbf{m}$ are expressed as

$$
\begin{aligned}
& \mathbf{g}=\left[\begin{array}{c}
\frac{\partial \mathrm{EI}}{\partial \rho} \\
\frac{\partial \mathrm{EI}}{\partial \phi} \\
\frac{\partial \mathrm{EI}}{\partial \mathbf{K}_{\mathrm{f}}}
\end{array}\right] \Delta \mathbf{d}, \\
& \mathbf{H} \approx \mathbf{g g}^{T},
\end{aligned}
$$

where $\mathbf{g}$ is the gradient of EI with respect to $\mathbf{m}, \mathbf{g}^{T}$ is the transpose of $\mathbf{g}, \Delta \mathbf{d}$ is the vector of difference between the inverted EI and the model EI, and $\mathbf{H}$ is the approximate Hessian matrix. To obtain the inverted density $\rho$, new parameterized porosity $\phi_{\mathrm{n}}$, and fluid bulk modulus $K_{\mathrm{f}}$, we use the full Newton step to compute the unknown parameter vector $\mathbf{m}$ as

$$
\mathbf{m}=\mathbf{m}_{0}+\gamma \Delta \mathbf{m}
$$

where $\mathbf{m}_{0}$ is the initial model of unknown parameter vector, which involves the initial models of $\rho, \phi_{\mathrm{n}}$, and $K_{\mathrm{f}}, \gamma$ is the step length, and $\Delta \mathbf{m}$ is the perturbation in $\mathbf{m}$, which is computed by

$$
\Delta \mathbf{m}=-\mathbf{H}^{-1} \mathbf{g}
$$

\section{Numerical Examples}

In this section, given a two-layer model, we first verify the accuracy of the simplified reflection coefficient, and then, we verify the robustness of the proposed inversion approach using the synthetic seismic data of signal-to-noise ratio (SNR) of 2. Real datasets acquired over a carbonate rock reservoir, which are well processed prior to being used in the inversion, are employed to further verify the reliability of the inversion approach.

3.1. Verification of Accuracy of the Simplified Reflection Coefficient. A two-layer model is utilized to verify the accuracy of the derived reflection coefficient. The minerals 
TABle 2: The computed P- and S-wave moduli of minerals and dry rock, new parameterized porosity, and fluid bulk modulus.

\begin{tabular}{lcccccccc}
\hline & $M_{0}(\mathrm{GPa})$ & $\mu_{0}(\mathrm{GPa})$ & $M_{\text {dry }}(\mathrm{GPa})$ & $\mu_{\text {dry }}(\mathrm{GPa})$ & $M_{\text {sat }}(\mathrm{GPa})$ & $P\left(\mathrm{~g} / \mathrm{cm}^{3}\right)$ & $\phi_{\mathrm{n}}$ & $K_{\mathrm{f}}(\mathrm{GPa})$ \\
\hline Layer 1 & 104.41 & 27.59 & 95.02 & 25.11 & 95.80 & 2.64 & 0.27 & 2.87 \\
Layer 2 & 103.12 & 27.22 & 78.57 & 20.74 & 78.60 & 2.50 & 0.71 & 0.044 \\
\hline
\end{tabular}

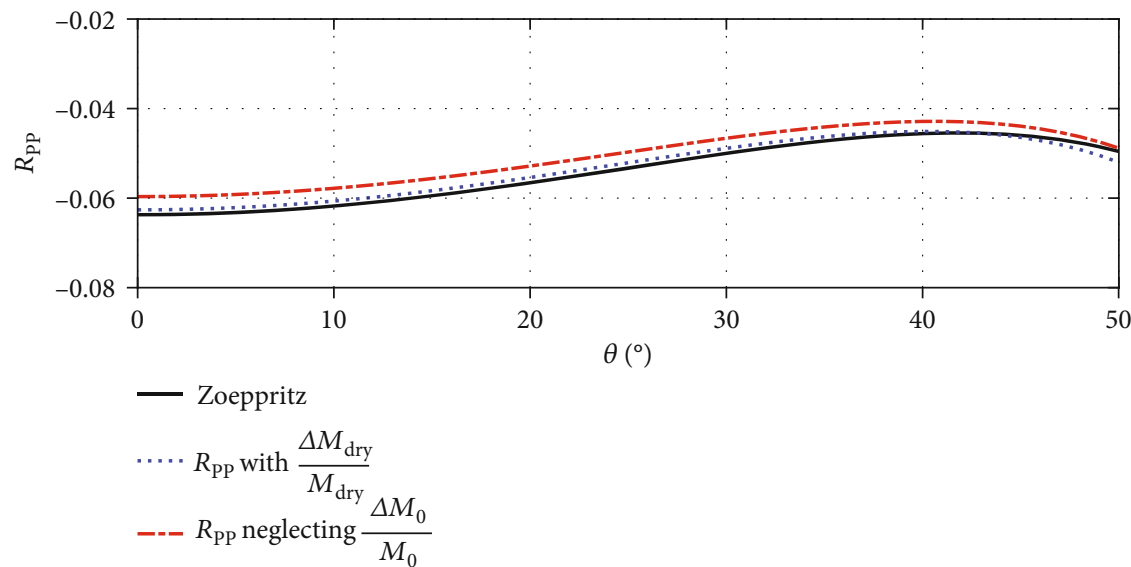

(a)

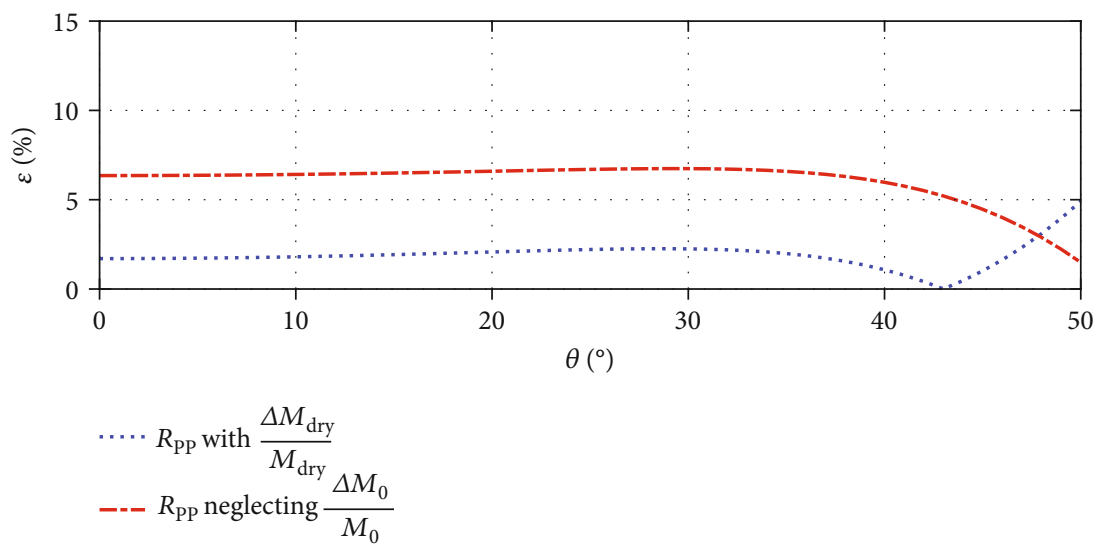

(b)

FIGURE 3: (a) Comparisons between results of $R_{\mathrm{PP}}$ computed using Zoeppritz equations, the derived reflection coefficient with $\Delta M_{\mathrm{dry}} / M_{\mathrm{dry}}$, and the simplified reflection coefficient neglecting $\Delta M_{0} / M_{0}$. (b) Relative error in the results computed using $R_{\mathrm{PP}}$ with $\Delta M_{\mathrm{dry}} / M_{\mathrm{dry}}$ and $R_{\mathrm{PP}}$ neglecting $\Delta M_{0} / M_{0}$, and the relative error is computed as $\varepsilon=\left|\left(R_{\mathrm{PP}}^{\text {derived }}-R_{\mathrm{PP}}^{\text {Zoepp }}\right) / R_{\mathrm{PP}}^{\text {Zoepp }}\right|$, in which $R_{\mathrm{PP}}^{\text {derived }}$ and $R_{\mathrm{PP}}^{\text {Zoepp }}$ represent results of $R_{\mathrm{PP}}$ computed using the derived reflection coefficient and Zoeppritz equations.

making up the rock are calcite and clay. Under the condition of small changes in lithology across the reflection interface, we assume change in the clay volume across the interface is small, and we also assume the fluid in pores is the mixture of gas and water. Table 1 shows the clay volume $V_{c}$, porosity $\phi$, and water saturation $S_{\mathrm{w}}$ for two layers, and Table 2 shows $\mathrm{P}$ - and S-wave moduli of minerals and dry rock, new parameterized porosity, and fluid bulk modulus. We stress that the effective P- and S-wave moduli of minerals are computed using the Voigt-Reuss-Hill average model presented by Mavko et al. [14], the P- and S-wave moduli of dry rock are calculated using equation (8), and the effective bulk modulus of fluids is computed using Wood's formula. The bulk moduli of calcite and clay are $76.8 \mathrm{GPa}$ and $25 \mathrm{GPa}$, and the shear moduli of calcite and clay are $32 \mathrm{GPa}$ and $9 \mathrm{GPa}$. The bulk moduli of water and gas are $2.87 \mathrm{GPa}$ and $0.04 \mathrm{GPa}$. The density of calcite, clay, water, and gas are $2.71 \mathrm{~g} / \mathrm{cm}^{3}, 2.55 \mathrm{~g} / \mathrm{cm}^{3}$, $1.0 \mathrm{~g} / \mathrm{cm}^{3}$, and $0.15 \mathrm{~g} / \mathrm{cm}^{3}$.

We next employ Zoeppritz equations, the derived reflection coefficient with $\Delta M_{\mathrm{dry}} / M_{\mathrm{dry}}$ (i.e., equation (7)), and the simplified reflection coefficient neglecting $\Delta M_{0} / M_{0}$ (i.e., equation (12)) to compute $R_{\mathrm{PP}}$ for the interface separating Layer 1 and Layer 2, and comparisons between the results of $R_{\mathrm{PP}}$ are shown in Figure 3.

We observe that there is a good match between results of $R_{\mathrm{PP}}$ computed using Zoeppritz equations and that calculated using the derived reflection coefficient with $\Delta M_{\text {dry }} / M_{\text {dry }}$ (i.e., equation (7)), and the relative errors computed using reflection coefficients that are calculated using $R_{\mathrm{PP}}$ neglecting $\Delta M_{0} / M_{0}$ and Zoeppritz equations are less than $10 \%$ in the case of the maximum 

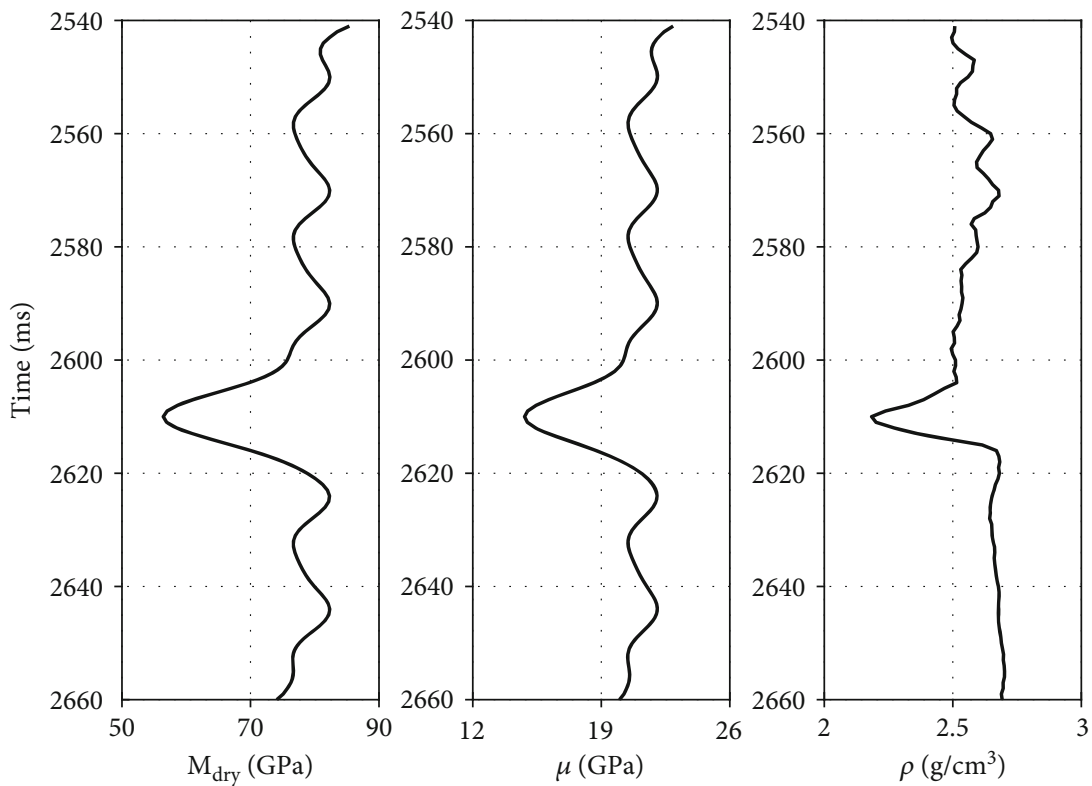

(a)
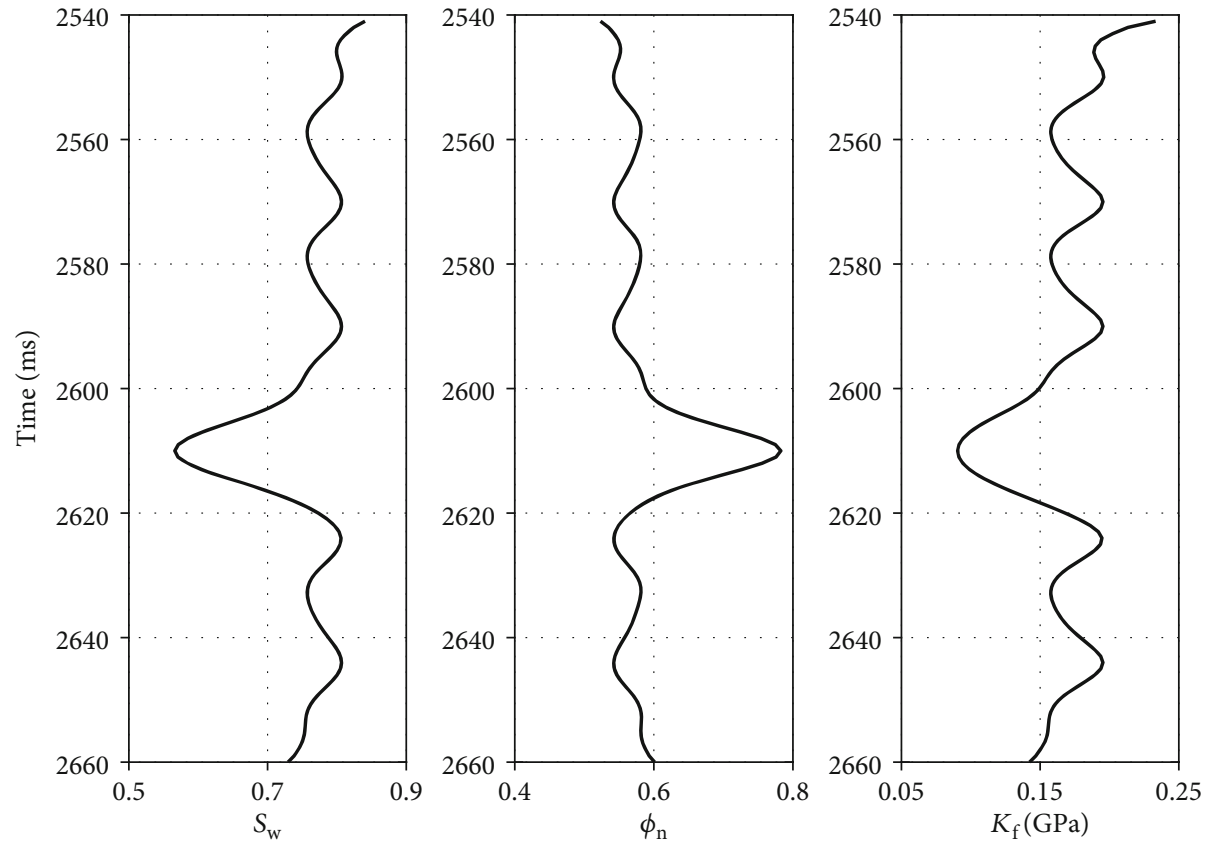

(b)

Figure 4: (a) Curves of P- and S-wave moduli of dry rock $\left(M_{\text {dry }}\right.$ and $\left.\mu\right)$ and density $\rho$ and (b) curves of water saturation $S_{\mathrm{w}}$, new parameterized porosity $\phi_{\mathrm{n}}$, and fluid bulk modulus $K_{\mathrm{f}}$.

incidence angle being around $30^{\circ}$, which reveals that the simplified reflection coefficient neglecting $\Delta M_{0} / M_{0}$ and $\Delta \mu_{0} / \mu_{0}$ can generate acceptable results of $R_{\mathrm{PP}}$ in the case of $\theta \leq 30^{\circ}$.

3.2. Robustness of the Proposed Inversion Approach. We utilize a model constructed using well log data (e.g., water saturation $S_{\mathrm{w}}$ and porosity $\phi$ ) to testify the robustness of the proposed inversion approach. Figure 4(a) plots curves of Pand S-wave moduli of dry $\operatorname{rock}\left(M_{\text {dry }}\right.$ and $\left.\mu\right)$ and density $\rho$, and Figure 4(b) plots curves of water saturation $S_{\mathrm{w}}$, new parameterized porosity $\phi_{\mathrm{n}}$, and fluid bulk modulus $K_{\mathrm{f}}$.
Using a Ricker wavelet of dominant frequency $30 \mathrm{~Hz}$, we generate synthetic seismic data employing results of $R_{\mathrm{PP}}$ computed using Zoeppritz equations and that calculated using the derived simplified reflection coefficient (i.e., equation (12)), as shown in Figure 5.

We observe that there is a good match between the synthetic seismic profiles generated using results of $R_{\mathrm{PP}}$ computed employing Zoeppritz equations and that calculated using the simplified reflection coefficient, which further verify the accuracy of the derived simplified reflection coefficient. 


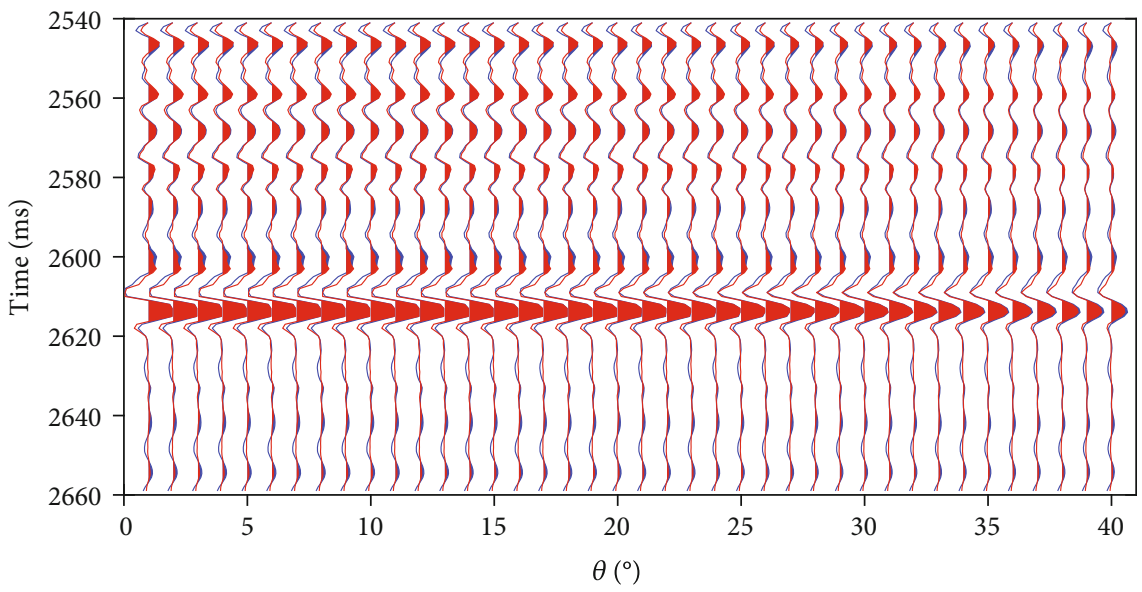

FIGURE 5: Comparison between synthetic seismic data generated using reflection coefficients computed employing Zoeppritz equations (blue) and the simplified reflection coefficient (red), respectively.
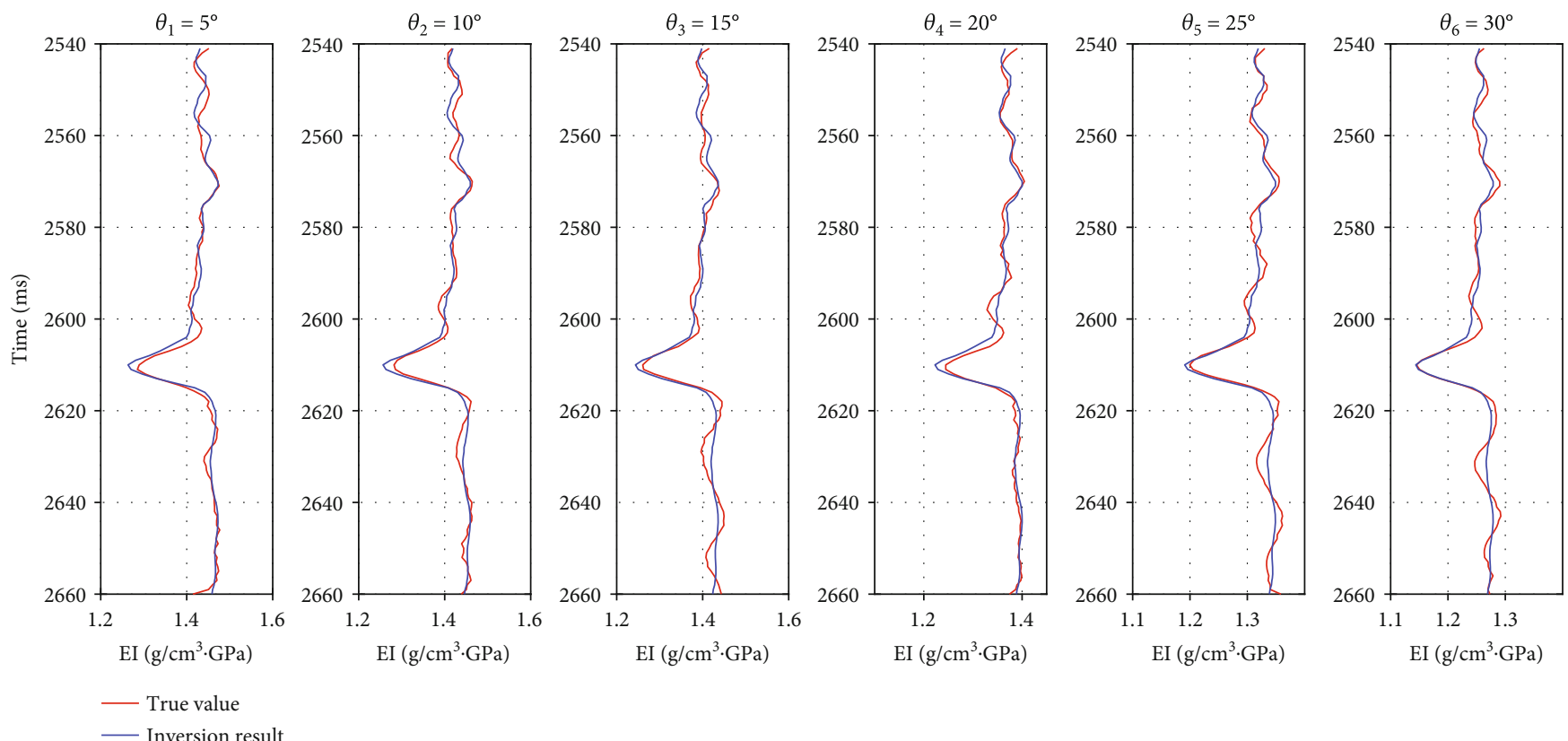

FIGURE 6: Comparisons between inversion results and true values of EI for the case of $\theta_{1}=5^{\circ}, \theta_{2}=10^{\circ}, \theta_{3}=15^{\circ}, \theta_{4}=20^{\circ}, \theta_{5}=25^{\circ}$, and $\theta_{6}=30^{\circ}$.

We next add Gaussian random noise to the synthetic seismic data that are generated using reflection coefficient computed using Zoeppritz equations in the case of $\theta_{1}=5^{\circ}$, $\theta_{2}=10^{\circ}, \theta_{3}=15^{\circ}, \theta_{4}=20^{\circ}, \theta_{5}=25^{\circ}$, and $\theta_{6}=30^{\circ}$ to obtain the noisy seismic data of SNR of 2. Prior to the inversion for $\mathbf{m}$ involving $\rho, \phi_{\mathrm{n}}$, and $K_{\mathrm{f}}$, we first estimate the result of EI from noisy seismic data using the LS algorithm. Comparisons between inversion results and true values of EI are shown in Figure 6. Using the estimated EI, we employ the proposed approach to implement the inversion for density, new parameterized porosity, and fluid bulk modulus.

In Figure 6, we observe that the EI datasets are estimated reliably from the noisy seismic data of SNR of 2, which means the estimated results of EI can be used for the second-step inversion for the unknown parameter vector $\mathbf{m}$. Comparison between the final inversion result and the true value of the unknown parameter is shown in Figure 7. We stress that the final inversion result is the average value computed using all the results estimated from the input EI datasets.

In Figure 7, we observe that the inversion result of unknown parameter can match the true value, which reveals the proposed inversion approach and workflow may produce reliable results of porosity and fluid bulk modulus for reservoir characterization and fluid discrimination.

3.3. Real Data Example. We next utilize real seismic data observed over a gas-bearing carbonate rock reservoir to further verify the reliability of the proposed inversion approach. We mention that the gas-bearing reservoir is embedded in carbonate rocks, which means the upper and lower rocks surrounding the reservoir are very similar. It also confirms that the derived reflection coefficient and the proposed inversion 

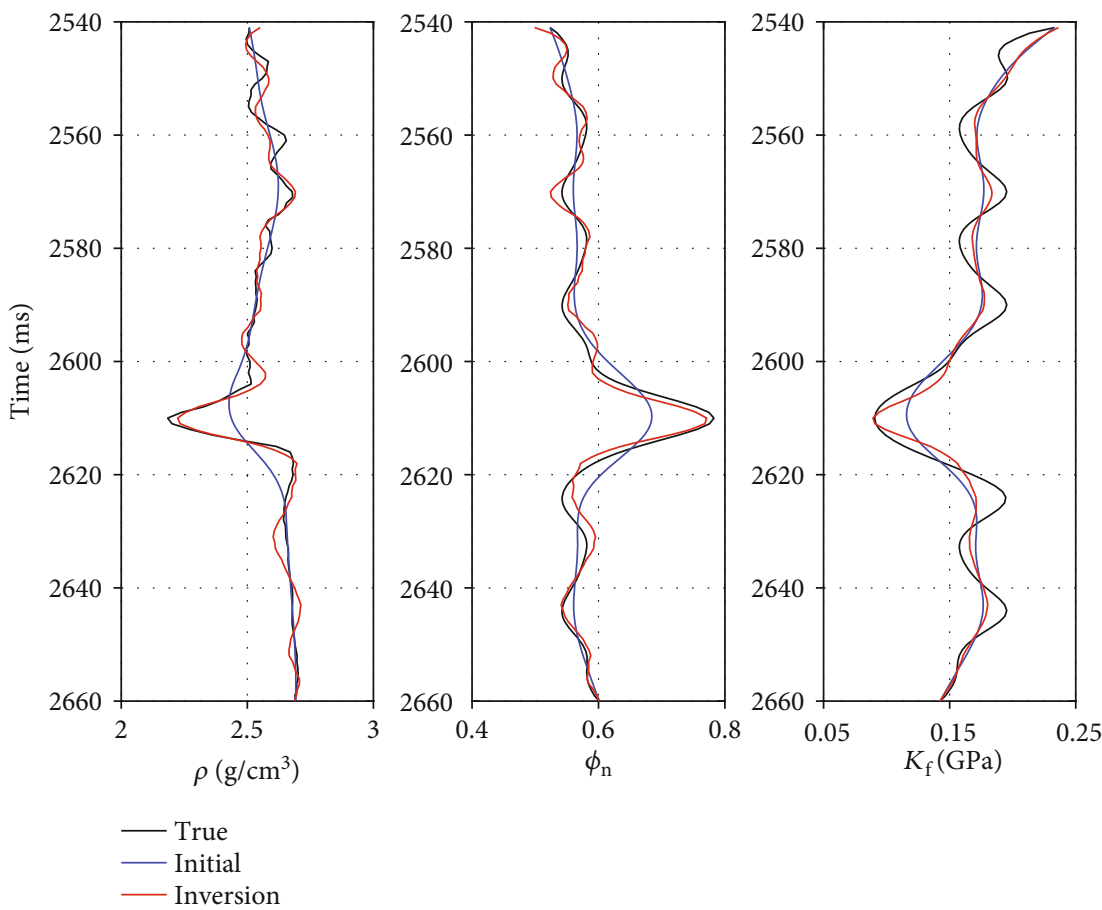

FiguRE 7: Comparison between true values (black) and inversion results (red) of density, new parameterized porosity, and fluid bulk modulus. Blue curves represent initial models of unknown parameters.
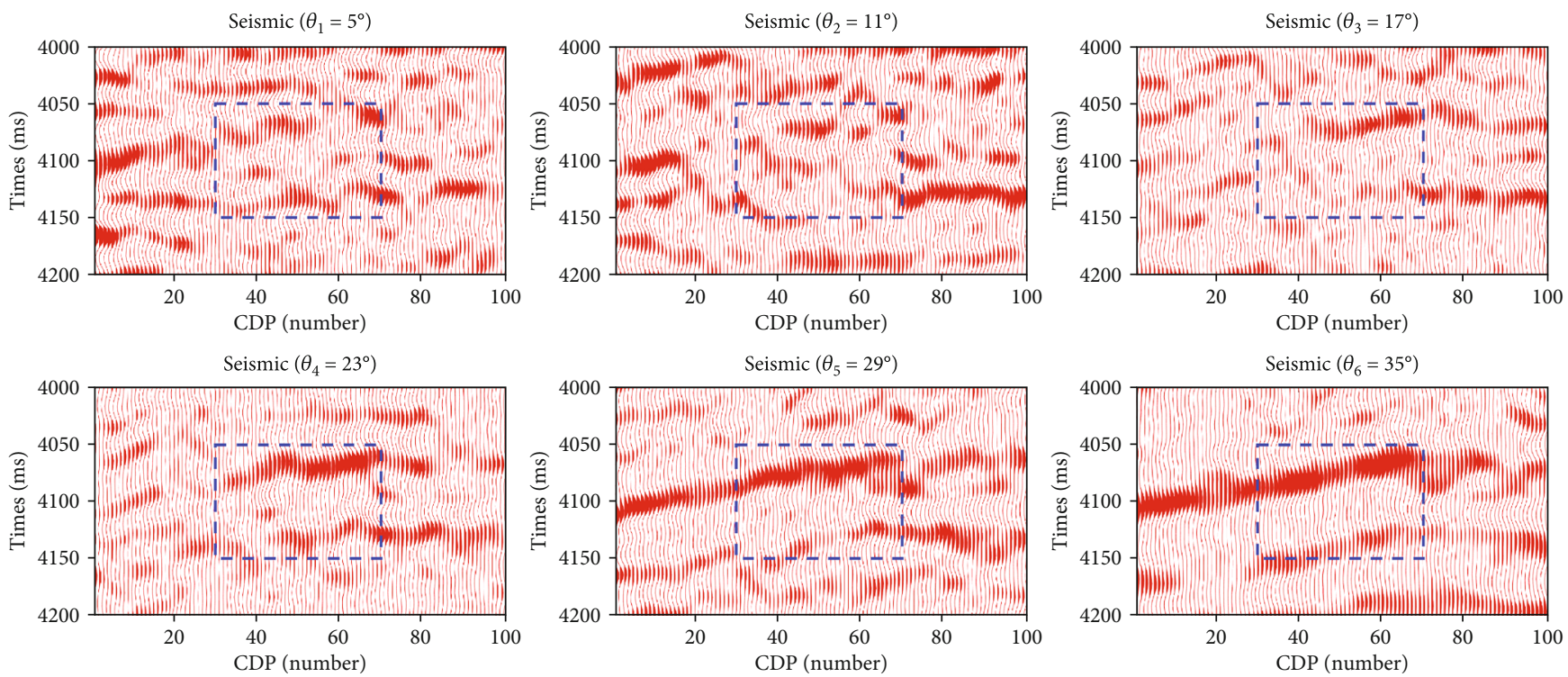

FIgURE 8: Stacked seismic data of different central angles. The dashed rectangle indicates the location of gas-bearing reservoir.

approach and workflow can be applied to this real seismic dataset. Figure 8 plots profiles of seismic data stacked over different ranges of incidence angle, i.e., seismic data of central angle $\theta_{1}=5^{\circ}$ are stacked using the data of angle range $2^{\circ}-7^{\circ}$; seismic data of central angle $\theta_{2}=11^{\circ}$ are stacked using the data of angle range $8^{\circ}-13^{\circ}$; seismic data of central angle $\theta_{3}$ $=17^{\circ}$ are stacked using the data of angle range $14^{\circ}-19^{\circ}$; seismic data of central angle $\theta_{4}=23^{\circ}$ are stacked using the data of angle range $20^{\circ}-25^{\circ}$; seismic data of central angle $\theta_{5}=29^{\circ}$ are stacked using the data of angle range $26^{\circ}-31^{\circ}$; and seis- mic data of central angle $\theta_{6}=35^{\circ}$ are stacked using the data of angle range $32^{\circ}-37^{\circ}$.

In Figure 8, we observe that the amplitude at the location of gas-bearing reservoir varies with the incidence angle (i.e., AVA phenomenon emerges). Using the LS algorithm proposed by Chen et al. [3], we first implement the inversion for EI. Figure 9 plots the inversion results of EI of different incidence angles. We observe that the inverted EI at the location of gas-bearing reservoir shows a relatively low value. 

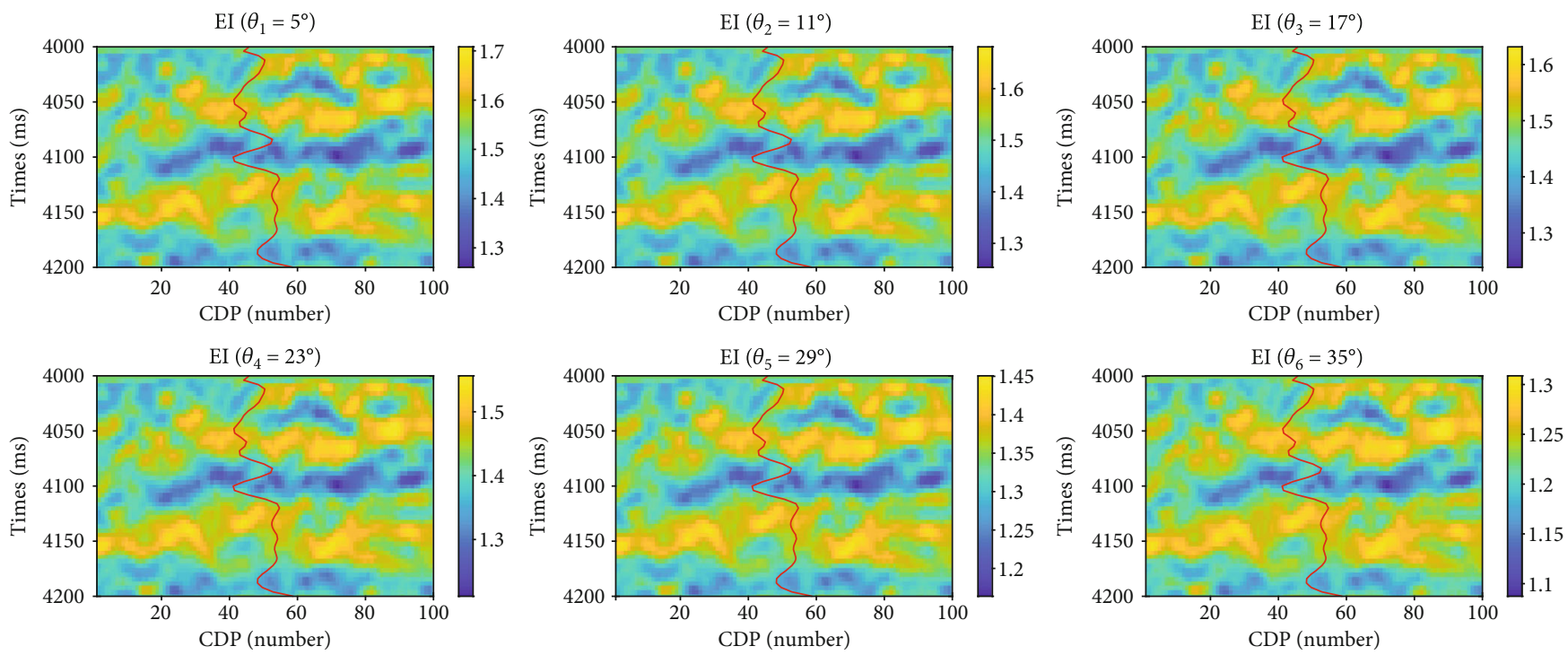

FIgURE 9: Inversion results of EI of different incidence angles. The curve indicates P-wave impedance computed using well log data.

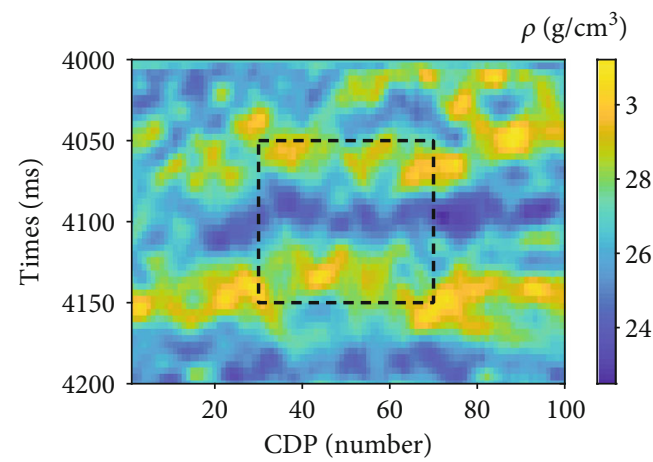

(a)

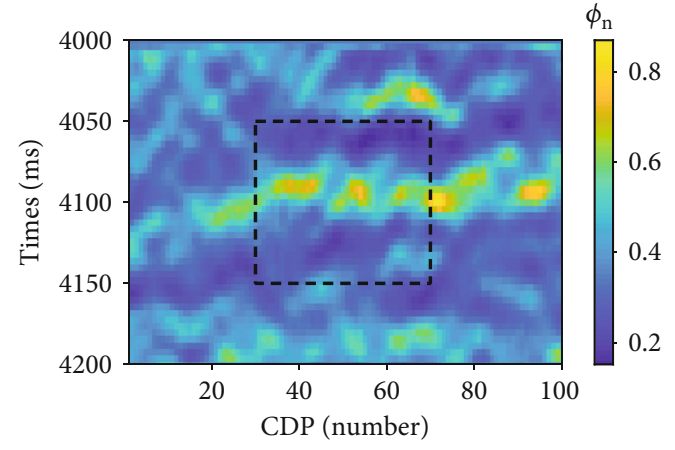

(b)

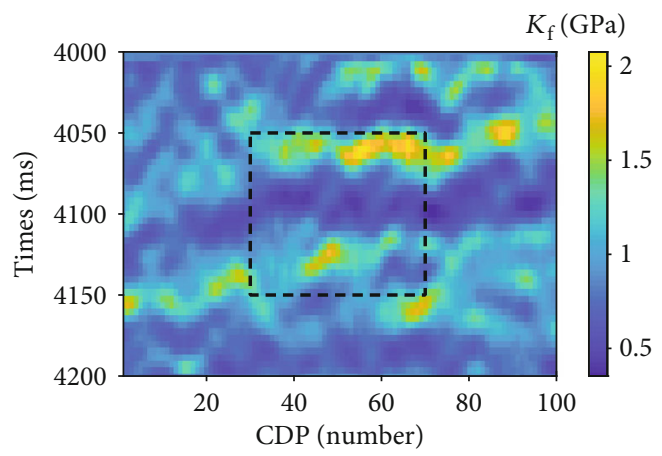

(c)

FIGURE 10: (a) Inversion result of density, (b) inversion result of new parameterized porosity, and (c) inversion result of fluid bulk modulus. The dashed rectangle indicates the location of gas-bearing reservoir.

Utilizing the inverted EI, we implement the estimation of unknown parameter vector $\mathbf{m}$ using the proposed inversion approach. In Figure 10, we plot the inversion results of density, new parameterized porosity, and fluid bulk modulus.

We observe that at the location of gas-bearing reservoir both the inversion results of density and fluid bulk modulus show relatively low values; however, the inverted new parameterized porosity shows a relatively high value. It reveals that the proposed inversion approach can provide reliable results of fluid bulk modulus and porosity, which is useful for fluid discrimination and reservoir characterization.

\section{Discussion}

In the present study, we present a linearized PP-wave reflection coefficient and elastic impedance (EI) in terms of moduli 

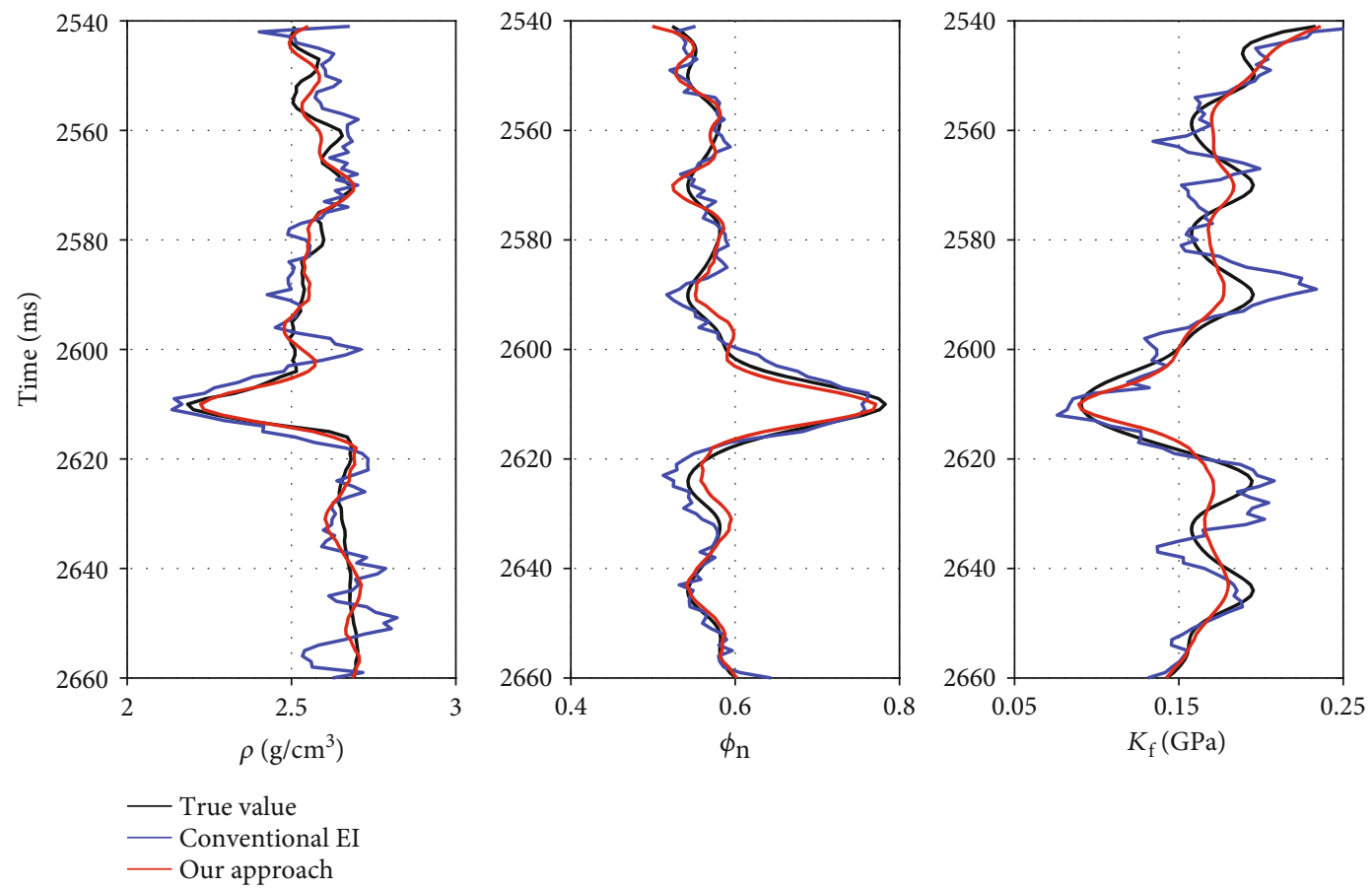

- Conventional E

FIGURE 11: Comparisons between inversion results obtained using our proposed inversion approach and that obtained using the conventional EI inversion.

of minerals and fluids and a new parameterized porosity. Based on the EI, we establish a two-step inversion approach of employing prestacked seismic data to estimate the new parameterized porosity and the fluid bulk modulus.

The advantage of the established two-step inversion approach is that we utilize the first- and second-order derivatives of elastic impedance with respect to unknown parameters to improve the accuracy of porosity inversion. Using the well log model shown in Figure 4, we implement the inversion for porosity and fluid bulk modulus using a conventional EI inversion approach following Zong et al. [10] and Chen et al. [4]. We explain that the conventional EI inversion approach is implemented as (1) the inversion of partially incidence angle-stacked seismic data for estimating EI of different dominant incidence angles, which is the same to the first step in our proposed inversion approach, and (2) the linear inversion of logarithmic EI for estimating elastic parameters (e.g., P- and S-wave moduli) and reservoir parameters (e.g., porosity and shale volume). However, in the second step of our proposed inversion, we employ the first- and second-order derivatives of EI with respect to elastic and reservoir parameters to implement the inversion for porosity and fluid bulk modulus, which may improve the accuracy of unknown parameter inversion. Here, we compare the results of porosity and fluid bulk modulus obtained using our proposed approach with those estimated using the conventional EI inversion approach, as shown in Figure 11. We observe that the result obtained using our inversion approach may match the true value better than that obtained using the conventional EI inversion method.

However, we should emphasize that assumptions under which we simplify the fluid substitution equations and derive the linearized reflection coefficient involve the following: (1) we employ the empirical relationship between $K_{\text {dry }}$ and $K_{0}$ proposed by Krief et al. [13], which is applicable to the case of $\phi \leq 0.2$; (2) we employ an approximately linear relationship between $\phi_{\mathrm{n}}$ and $\phi$ in the case of $\phi \leq 0.1$; (3) the accuracy of the derived reflection coefficient is verified in the case that the maximum incidence angle is around $30^{\circ}$; (4) we neglect the effect of moduli of minerals on the reflection coefficient in the case that differences between bulk and shear moduli of minerals making up rocks of the upper and lower layers are small. In conclusion, the derived reflection coefficient and proposed inversion approach are applicable to reservoirs embedded in rocks with the same lithology of $\phi \leq 0.1$, and seismic data of maximum incidence angle being around $30^{\circ}$ are employed to implement the inversion.

\section{Conclusion}

Starting with the model of fluid substitution, we first propose a simplified bulk modulus of saturated rock, in which we employ a new parameterized porosity to rewrite the bulk modulus of dry rock. Using the simplified bulk modulus, we derive PP-wave reflection coefficient in terms of reflectivities of moduli of fluids and minerals and new parameterized porosity. Focusing on the case of reservoirs embedded in rocks with the same lithology, we further simplify the derived reflection coefficient. Based on the derived reflection coefficient, we establish an approach of employing prestacked seismic data to implement the inversion for new parameterized porosity and fluid bulk modulus. Tests on noisy synthetic seismic data verify the stability of the proposed inversion approach, and applying the proposed approach to real data 
acquired over a gas-bearing carbonate reservoir verifies that the inversion approach can provide reliable results of fluid bulk modulus and porosity, which is useful for fluid identification and reservoir characterization.

\section{Data Availability}

Well logging curves and real datasets are provided by PetroChina. We have signed an agreement of data use with PetroChina, and the data cannot be released.

\section{Conflicts of Interest}

The authors declare that they have no conflicts of interest.

\section{Acknowledgments}

This research work is supported by the National Science and Technology Major Project (no. 2016ZX05007-006) and the Major Project of PetroChina (no. 2019B-0607). We thank Dr. Junxiao Li for his help in improving the writing and editing of this paper.

\section{References}

[1] F. Gassmann, "Über die elastizität poröser medien," Vierteljahrsschrift der Naturforschenden Gesellschaft Zürich, vol. 96, pp. 1-23, 1951.

[2] K. Aki and P. G. Richards, Quantitative Seismology, University Science Books, 1980.

[3] H. Chen, J. Li, and K. A. Innanen, "Nonlinear inversion of seismic amplitude variation with offset for an effective stress parameter," Geophysics, vol. 85, no. 4, pp. R299-R311, 2020.

[4] H. Chen, Y. Ji, and K. A. Innanen, "Estimation of modified fluid factor and dry fracture weaknesses using azimuthal elastic impedance," Geophysics, vol. 83, no. 1, pp. WA73-WA88, 2018.

[5] J. L. Fatti, G. C. Smith, P. J. Vail, P. J. Strauss, and P. R. Levitt, "Detection of gas in sandstone reservoirs using AVO analysis: a 3-D seismic case history using the Geostack technique," Geophysics, vol. 59, no. 9, pp. 1362-1376, 1994.

[6] B. Goodway, T. Chen, and J. Downton, "Improved AVO fluid detection and lithology discrimination using Lamé petrophysical parameters; " $\lambda \rho$ ", " $\mu \rho$ ", \& " $N \mu$ fluid stack", from P and S inversions: 67th Annual International Meeting," in SEG, Expanded Abstracts, pp. 183-186, Dallas, Texas, USA, 1997.

[7] B. H. Russell, D. Gray, and D. P. Hampson, "Linearized AVO and poroelasticity," Geophysics, vol. 76, no. 3, pp. C19-C29, 2011.

[8] B. H. Russell, K. Hedlin, F. J. Hilterman, and L. R. Lines, "Fluid-property discrimination with AVO: a Biot-Gassmann perspective," Geophysics, vol. 68, no. 1, pp. 29-39, 2003.

[9] X. Yin and S. Zhang, "Bayesian inversion for effective porefluid bulk modulus based on fluid-matrix decoupled amplitude variation with offset approximation," Geophysics, vol. 79, no. 5, pp. R221-R232, 2014.

[10] Z. Zong, X. Yin, and G. Wu, "Elastic impedance parameterization and inversion with Young's modulus and Poisson's ratio," Geophysics, vol. 78, no. 6, pp. N35-N42, 2013.

[11] P. Connolly, "Elastic impedance," The Leading Edge, vol. 18, no. 4, pp. 438-452, 1999.
[12] J. E. Downton, Seismic Parameter Estimation from AVO Inversion, Ph.D. thesis, University of Calgary, 2005.

[13] M. Krief, J. Garat, J. Stellingwerf, and J. Ventre, “A petrophysical interpretation using the velocities of $\mathrm{P}$ and $\mathrm{S}$ waves (fullwaveform sonic)," The Log Analyst, vol. 31, pp. 355-369, 1990.

[14] G. Mavko, T. Mukerji, and J. Dvorkin, The Rock Physics Handbook: Tools for Seismic Analysis of Porous Media, Cambridge University Press, 2009. 